\title{
Capacity of Pervasive Camera Based Communication Under Perspective Distortions
}

\author{
Ashwin Ashok, Shubham Jain, Marco Gruteser, Narayan Mandayam, Wenjia Yuan, Kristin Dana \\ WINLAB, Rutgers University, 671 Route 1 South, North Brunswick, NJ, USA \\ Email:\{aashok@winlab, shubhamj, gruteser@winlab, narayan@winlab, wenjiay, kdana@ece\}.rutgers.edu
}

\begin{abstract}
Cameras are ubiquitous and increasingly being used not just for capturing images but also for communicating information. For example, the pervasive $Q R$ codes can be viewed as communicating a short code to camera-equipped sensors and recent research has explored using screen-to-camera communications for larger data transfers. Such communications could be particularly attractive in pervasive camera based applications, where such camera communications can reuse the existing camera hardware and also leverage from the large pixel array structure for high data-rate communication. While several prototypes have been constructed, the fundamental capacity limits of this novel communication channel in all but the simplest scenarios remains unknown. The visual medium differs from RF in that the information capacity of this channel largely depends on the perspective distortions while multipath becomes negligible. In this paper, we create a model of this communication system to allow predicting the capacity based on receiver perspective (distance and angle to the transmitter). We calibrate and validate this model through lab experiments wherein information is transmitted from a screen and received with a tablet camera. Our capacity estimates indicate that tens of Mbps is possible using a smartphone camera even when the short code on the screen images onto only $15 \%$ of the camera frame. Our estimates also indicate that there is room for at least $2.5 \mathrm{x}$ improvement in throughput of existing screen - camera communication prototypes.
\end{abstract}

\section{INTRODUCTION}

The pervasive use of cameras has led to not only a diverse set of camera-based sensing applications but also to novel opportunities to use cameras to communicate information [10]. Recent efforts to standardize camera communications [3] attests to the importance of using camera for communications. Camera based communication is characterized by highly directional transmission and reception along with low-multipath interference rendering it virtually interference-free. Thus, it is particularly attractive for dense congested environments where RF communication data rates are largely limited due to interference, for security applications where the directional transmissions lead to lower probability of interception or observability of signals, or for situations where the high directionality leads to improved localization of the transmitters. Camera based communication can leverage existing cameras for communicating with the ubiquitous light emitting devices. Information could be transmitted from TVs, monitors, billboards, and even projector screens. We believe, therefore, that camera-based communications can be an attractive alternative or supplement to RF wireless based communication.

Today, cameras are frequently used to read QR-codes, which can be considered as a form of visual communication wherein the camera acts as a receiver. The ubiquitous use of QR codes motivates building novel camera communication applications, where pervasive display screens could be modulated to send time-varying QR codes to be decoded by video cameras. The large pixel array elements of the screen and camera can be leveraged to send high volume of data through short time-varying 2D barcodes. For example, a user could point a camera to a desktop PC or even a smartphone screen displaying the time-varying code to download a file or perhaps a video. Recent research has further explored this direction by designing prototypes wherein time-varying $2 \mathrm{D}$ barcodes can be transmitted from desktop monitors [17], [20] and smartphone screens [14] to a camera receiver. While these works have designed and measured the performance of specific point solution in this space, how much room for improvement exists in these solutions or if there is any bound on performance still remains unclear.

QR-code recognition is typically limited to short distances of $\mathrm{cm}$ and the camera usually has to be well-aligned so that the code covers most of the camera image. The throughput is largely affected by any change in perspective (position or orientation) of the camera with the transmitter. When the camera is far from the screen (or at a highly oblique angle) light rays from multiple transmitter elements (pixels of the screen) interfere on one or more camera pixels, causing interpixel interference (IPI), reducing received signal quality and throughput.

To our knowledge, only few projects have begun to investigate information capacity limits of camera communication using time-varying 2D barcodes. Hranilovic et.al. [17] analyzed the capacity and prototyped a screen-camera system where a CCD camera was placed at a fixed distance from a laptop screen. The model does not account for the interference between pixels and the dependence on perspective. The model in [10] can be considered as a simplified case of screen-camera channel where the transmitter and receiver always remain aligned, while ignoring the quantization effects of real camera receivers.

In this paper, we develop a model for the information capacity of screen-camera communication that accounts for perspective dependent (position and orientation) distortions that dominate this channel. The model incorporates projection theory from the computer vision domain into a Shannon capacity formulation. Specifically, our contributions in this 
paper are:

- A screen-camera channel model that accounts for perspective distortions and realities of camera receivers such as quantization limitations.

- Experimental calibration and validation of the model through extensive lab measurements using a screen transmitter and a tablet camera receiver. The studied parameters include distance, angle, and granularity or block-size of the code (number of pixels per transmit bit).

- Estimation and validation of capacity for screen-camera communication by measuring channel and signal quality metrics, such as bandwidth and signal-to-interferencenoise ratio, and substituting into the derived analytical capacity expression.

- A comparison of capacity estimate with throughput of existing screen-camera communication prototypes.

\section{BACKGROUND ON CAMERA COMMUNICATION}

Camera based communication is a class of visible light communications (VLC) [8], where information is modulated through light transmitted from optical emitters such as LEDs and LCDs, and received by photo-receptor elements at the receiver (in a camera, the image sensor pixels are the photoreceptors). The inherent $2 \mathrm{D}$ spatial array structure of the image sensor pixels can be leveraged to create a multi-input-multioutput (MIMO) channel by using arrays of optical emitter elements to transmit information through a concept called visual MIMO [10]. In this regard, the array of LEDs in lighting arrays and commercial display devices, LCD pixels in display screen, projector screens, or printed material ${ }^{1}$ qualify as potential transmitters in camera based communications.

A camera channel is analogous to a RF MIMO channel where each pixel element of the camera acts as a receiving antenna and the light emitting elements as the transmit antennas. In RF MIMO, the signal quality at each receive antenna element is a function of the path-loss in the channel, multipath fading, and the interference from other transmit antennas also called co-channel interference [7]. A camera channel has negligible multipath fading but experiences path-loss in light energy, and interference (of light energy) from other light emitting elements, which manifest as visual distortions on the output of a camera, that is, the image. These distortions can be modeled (deterministically) using classical camera imaging theory.

The signal quality at the camera receiver is also influenced by noise in the channel. Noise in camera systems manifests as noise current on each camera pixel, and is generated due to the photons from, environment lighting (includes ambient lighting) and from the transmitter and receiver imaging circuitry [19]. Noise current in a pixel is usually considered signal independent when the ambient lighting is sufficiently high compared to the transmit signal; for example, in office rooms or outdoors [21]. At the output of a camera, the noise current

\footnotetext{
${ }^{1}$ Barcodes such as QR codes printed on papers qualify as time-invarying messages
}

in each camera pixel is a quantized quantity and manifests as fluctuations in the intensity (digital value of the sensor output) of that pixel; the noise energy accumulated in each pixel can be quantified using the mean value of variance in the pixel intensity. As in prior works on modeling optical channels [21], [23], in this paper, we will consider that the noise in a camera pixel is primarily from background, and follows a AWGN characteristic, and is uniform over the image sensor (photoreceptor); quantified through the AWGN noisevariance $\sigma_{n}^{2}$.

Considering the deterministic nature of perspective distortions and the AWGN channel, capacity (measured in bits/sec) of camera based communication can be expressed using Shannon Capacity formula as,

$$
C=W_{f p s}\left(W_{s} \log _{2}(1+S I N R)\right)
$$

where $S I N R$ represents the signal-to-interference-noise ratio per pixel, $W_{f p s}$ is the camera-frame rate or the receiver sampling rate in frames-per-second. $W_{s}$ is the spatialbandwidth, which denotes the number of information carrying pixels per camera image frame. The spatial bandwidth is equivalent to the number of orthogonal or parallel channels in a MIMO system. In the rest of the paper, we will use the terms screen transmitter and screen interchangeably, and the term image to refer to the camera sampled image.

\section{SCREEN - CAMERA CHANNEL}

In screen-camera communication, information is modulated in the light intensity of the pixel elements of a screen transmitter that are received and decoded from the camera image pixel intensity at the receiver. The pixel intensity in a camera image is a digital quantity ${ }^{2}$ that is proportional to the amount of photon current generated on the pixel from the light energy accumulated over its area (the smaller the pixel area the lesser light intensity it accumulates). When the light emitting screen pixel is at the focus of the camera lens (and hence the camera pixel) all the light rays from the screen pixel are focused onto the camera pixel and thus incurring no loss of energy on the pixel. When the screen pixel is perturbed (in position and/or orientation) from the focus of the camera or incurs path-loss in energy, due to the finite aperture size of the camera lens, not all light rays converge on the camera pixel resulting in reduced accumulated energy and hence a smaller pixel intensity value. The loss in the received light intensity on a camera pixel results in the visual deformation in size or shape of the imaged screen pixel; an effect that is termed as perspective distortion.

Loss in signal energy on a pixel is also attributed to the noise in that pixel. As discussed earlier, noise in a camera pixel is primarily due to photons from the environment, which can be modeled as signal independent and AWGN. Noise from the transmitter and the camera imaging circuit are dependent on the generated signal (and that is transmitted), and thus depend on the transmitter and receiver specifications. However, unlike

\footnotetext{
${ }^{2}$ most cameras have 8 bit monochromatic depth (on each colour channel) where the values span 0 (dark)-to-255 (bright)
} 


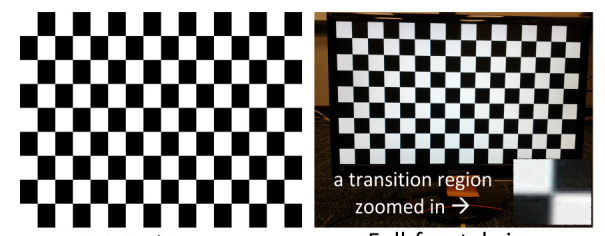

Original Image

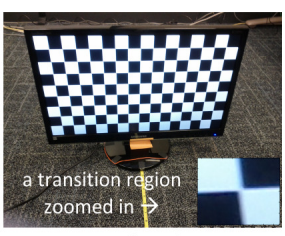

Angular view
Fig. 1. Illustration of perspective distortion in screen-camera channel. Imaged screen pixels are blurry, and reduced in size in full-frontal view and also in shape in angular view.

environment noise, this signal dependent noise can be estimated using one-time calibration mechanisms; camera noise modeling has been well studied in computer vision and solidstate electronics (CMOS) design literature. We reserve the discussions on effect of signal dependent noise on throughput of camera communications for future work.

Perspective Distortions. Distortions that depend on the perspective of the camera are caused due to the nature of the camera imaging mechanism and manifest as deformation in size and shape of the captured object (the light emitting screen pixel) on the image, resulting in visual compression or magnification of the object's projection on the image. When the screen is at an out-of-focus distance from the camera lens (or at an oblique angle), these distortions become prominent and lead to interference between adjacent screen pixels on the camera image, what we term as inter-pixel interference or IPI. The combined effect of background noise and IPI degrades the received signal quality and hence reduces information capacity in camera channels. For example, let us consider that blocks of screen pixels are illuminated by a chessboard pattern and imaged by a camera as shown in Fig. 1. We can observe that perspective distortions cause the screen pixels to deform in size when the screen is not at the focus of the camera, and in shape when it is not frontally aligned (viewed at an angle) with the camera. If the screen pixel was at the focus, and assuming the screen and camera have the same resolution, it's image on the camera should occupy the same area as one pixel. But in reality, the light rays from the screen pixel may not end exactly on camera pixel boundaries and there is some area surrounding it that accumulates interference. This area of misalignment and the geometry of the imaged screen pixel will be perspective dependent and accounts for distortion due to perspective scaling of the pixel area.

We can also observe from Fig. 1 that the imaged screen pixels are blurry, especially at the transition regions between white and black blocks. This blur effect is attributed to the camera lens and more formally termed as lens-blur. It is modeled by the point-spread function (PSF) in computer vision theory [16], which represents the response of an imaging system to a point source. Lens-blur causes the received light energy to spread to areas outside the pixel, where the amount of spread depends on the type of lens being used. Lensblur can be understood as a low-pass filtering phenomenon that distorts the high-frequency components in the image, such as edges and high contrast regions [13]. In the screen-

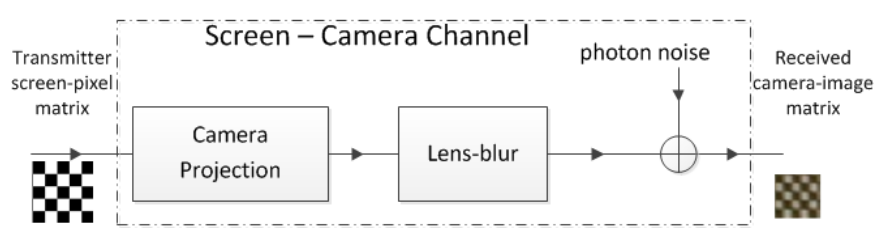

Fig. 2. Screen - Camera Channel Model

camera channel this translates to distorting the pixels at the transition regions between brighter (high intensity) and darker (low intensity) pixels, and leads to interference (IPI) between neighboring pixels, as seen in Fig. 1. Since the area and the maximum energy that can be sampled in each camera pixel is finite, IPI leads to an effective reduction in signal energy per pixel. Unlike fading in RF wireless channels, distortions in camera channels are deterministic, and can be modeled using camera imaging theory, as exercised in this paper.

\section{A. Perspective Distortion Factor}

We model the perspective distortions in the screen-camera channel as a composite effect of signal energy reduction due to perspective scaling of pixel area owing to camera projection, signal energy reduction due to lens-blur, and background photon noise, as shown in Fig. 2. In this regard, let us consider that the signal energy on each pixel is weighted by perspective distortion factor $\alpha$, that represents the effective area scaling (down) due to perspective and lens-blur in the camera imaging process, while the rest of the light-energy on the pixel is from ambient photon noise. We define this factor such that it can take values in $0 \leq \alpha \leq 1$, where $\alpha=1$ indicates that the screen pixel is at the focus of the camera and also incurs no signal reduction due to lens-blur, and $\alpha=0$ indicates that no part of the screen-pixel gets imaged on the camera pixel.

As discussed earlier, lens-blur causes the signal energy to leak outside the area of a single pixel. Camera lens-blur, characterized by the PSF, can be approximately modeled as a 2D gaussian function [6], [16], where the amount of spread in area is quantified using its variance $\sigma_{b l u r}^{2}$ (a large variance indicates more blur ${ }^{3}$ ). In our model we account for lens-blur distortion using the factor $\alpha_{b}=\left(2 \sigma_{b l u r}\right)^{2}$, to account for the spread in area over two dimensions of the square pixel. If $s_{c a m}$ is the side length of a camera pixel, then the effective signal energy on that pixel will be proportional to $s_{\text {cam }}^{2} \frac{1}{1+\alpha_{b}}$. We treat this signal energy reduction is proportional to this reduced pixel area over which the signal accumulates.

Let $\alpha_{p}$ represent the perspective scaling of the area of an imaged screen pixel when perturbed from camera focus. We model this perspective scaling factor and derive a general expression for $\alpha_{p}$ in Appendix A using camera projection theory [15], that uses the camera projection matrix which maps the location of the screen pixels from the world coordinate frame to the camera coordinate system. In the simplest case,

\footnotetext{
${ }^{3}$ For an ideal pin-hole camera energy spread over a pixel would be uniform and hence $\sigma_{b l u r}^{2}$ is infinitesimally small
} 


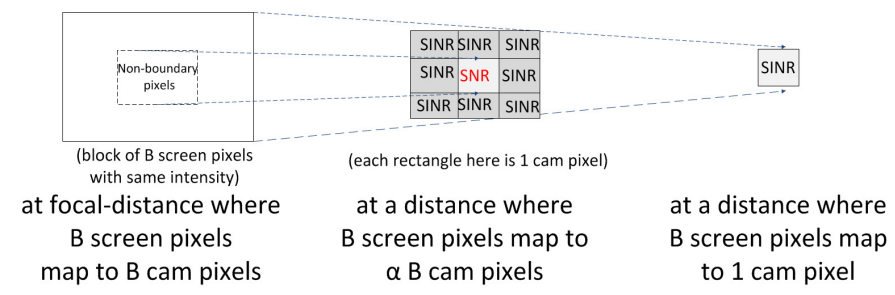

Fig. 3. Illustration of interference between pixel-blocks due to perspective distortion for SINR computation

where the screen and camera are perfectly aligned at distance $d$, this factor can be expressed as,

$$
\alpha_{p}=\left(\frac{f_{c a m} s_{t}}{s_{c a m} d}\right)^{2}
$$

where $f_{c a m}, s_{t}$ are the focal length of the camera and side-length of the screen pixel, respectively. As can be seen from equation 2 (or 16), $\alpha_{p}$ is a function of the distance and angle between the screen and camera. We can observe from equation (2) that, $\alpha_{p}=1$ when the camera is at the focus $\left(d=f_{\text {cam }}\right)$ and if $s_{c a m}=s_{t}$. However, in reality, the physical size of a screen and camera pixel may not be the same. In our system, we assume that the focal point is at a distance $d_{f}=\frac{f_{c a m} s_{t}}{s_{c a m}}$ to the screen; which we term as focal-distance.

If $\alpha$ denotes the average distortion in each pixel of the camera image, we express $\alpha$ as the effective pixel area reduction due to perspective scaling factor $\alpha_{p}$ on the reduced pixel area due to lens-distortion $\alpha_{b}=4 \sigma_{b l u r}^{2}$, as

$$
\alpha=\alpha_{p} \times \frac{1}{\left(1+\alpha_{b}\right)}
$$

\section{B. Signal-to-Interference Noise Ratio}

We quantify the quality of the signal at the camera receiver in the screen-camera channel using the average SINR per pixel,

$$
S I N R_{\alpha}=\frac{\alpha P_{a v g}^{2}}{(1-\alpha) P_{a v g}^{2}+\sigma_{n}^{2}}
$$

where, $P_{\text {avg }}$ denotes the average transmit pixel intensity (for example, a screen-camera system using black (0) and white (255) intensities for transmission will have $P_{\text {avg }}=127.5$ ). By using the digital value of the average signal $P_{a v g}$, instead of its analog equivalent (pixel photon-current squared), our model accounts for the quantization limitations in cameras. The $1-\alpha$ term in equation (4) quantifies the fraction of the pixel area affected by interference. $\sigma_{n}^{2}$ denotes average AWGN noise energy in each pixel, and since noise uniformly affects the entire area the pixel it does not depend on the scaling factor $\alpha$.

Pixel blocks. A small value of $\alpha$ indicates that more screen pixels interfere on one camera pixel. In reality, screen pixels are very closely spaced (fraction of a $\mathrm{mm}$ ), and so, IPI will be inevitable even at short distances (since $\alpha_{p}$ is very small as $s_{t}$ is very small) resulting in low SINRs. A potential solution is to leverage the MIMO structure of the screen-camera channel, by grouping multiple screen pixels in a block, such as a $2 \mathrm{D}$ barcode, to transmit with same intensity, and combine those imaged pixels on the camera receiver to improve SINR. This technique, in principle, is similar to diversity combining used in RF MIMO. Pixel-blocks merely represent that a group of antennas are used to transmit the same intensity, to improve the SINR at the receiver. By using pixel blocks, we draw analogies of the screen-camera channel to an equivalent MIMO system, and not multiple-level modulation or coding. Since channel capacity is essentially independent of the type of modulation or coding used, we reserve our discussions on modulation and coding schemes for screen-camera communication for future.

Misalignment of Pixels and Blur. Mismatch between the physical size of screen display and camera pixels can cause an imaged screen pixel not to align with a camera pixel, even if the screen pixel were at the camera focus. Such misalignments will cause a deviation in the distortion factor for each pixel as the perspective changes. However, such deviations can be assumed to be negligible when considering an average distortion factor over the camera image. Though, one can assume some vibrations on the pixels, especially when the camera is not stable, which means that the area of misalignment can keep changing with perspective. Such dynamic change in perspective arises primarily when the camera is hand-held, due to hand-shakes or lateral movements; it also applies for many more stationary scenarios. In such cases, the distortion effect seen is in the form of blurry and mixed frames due to motionblur; the blur effect arises due to movement within or between camera frames, and has been well studied in computer vision literature [16]. The impact of misalignments, and also lensblur, will become smaller as one block covers more pixels on the camera and only affect pixels near the boundary as shown in Fig. 3. As a convention in our model, we treat a pixel block as a boundary block if it is not all surrounded by blocks with same intensity. Such a structure minimizes the 'interference'for a non-boundary pixel, and is negligible when the camera and screen are static with respect to each other. In this case, even for a non-zero blur or pixel misalignment, since the same signal adds-up on the pixel, it enhances signal energy of that pixel; in which case the SINR of that pixel converges to the average-SNR.

In general, the expression for the average SINR per imaged block in a screen-camera channel, using B pixel square blocks of a screen can be given as,

$$
\begin{array}{rlrl}
S I N R_{b l k}(\alpha, B) & =\gamma_{1} S I N R_{\alpha}+\gamma_{2} S N R_{\alpha} \forall \alpha B>4 \\
& =S I N R_{\alpha} & \forall \alpha B \leq 4
\end{array}
$$

where $S I N R_{\alpha}$ is from equation $4, S N R_{\alpha}=\frac{\alpha P_{a v g}}{\sigma_{n}^{2}}$, and the coefficients $\gamma_{1}=4(\sqrt{\alpha B}-1)$ and $\gamma_{2}=(\sqrt{\alpha B}-2)^{2}$ represent the number of boundary-blocks and non-boundary blocks, respectively. We consider that $\min B=4$ (i.e. $2 \times 2$ pixels), and $\alpha B \leq 4$ indicates that each $\mathrm{B}$ pixel block projects onto a maximum of 1 camera pixel area and $\alpha B>4$ indicates 
that the block projects onto multiple camera pixels.

\section{Capacity Under Perspective Distortions}

Recalling the capacity expression from equation (1), we can express the capacity of screen-camera communication in bits/sec as,

$$
C_{\text {cam }}(\alpha)=\frac{W_{f p s}}{2} \alpha\left\|R_{\text {cam }}\right\| \log _{2}\left(1+\operatorname{SIN} R_{\alpha}\right)
$$

where $S I N R_{\alpha}$ is the signal-to-interference noise ratio from equation (4), $\left\|R_{\text {cam }}\right\|$ denotes resolution of the camera and $W_{f p s}$ denotes the frame-rate of the camera in frames-persecond. The camera frame-rate, and hence bandwidth, is halved (following Nyquist sampling theory) to avoid the mixed frames caused by aliasing resulting from the synchronization mismatch between screen updates and the camera sampling. The term $\alpha\left\|R_{\text {cam }}\right\|$ represents the total number of camera pixels that contain the image of the screen pixels, and is essentially the spatial-bandwidth term $W_{s}$ in equation 1 . This is very different from RF MIMO, where, all the receiver antennas can potentially receive the signal, independent of distance between the transmitter and receiver. In a camera receiver, due to its LOS nature, the signal from each transmit element is always limited to a finite number of, but never all, receive elements.

The capacity in equation 6 represents the upper bound on the total number of bits that can be communicated with negligible error from 1 screen pixel to a camera pixel. Grouping pixels into blocks improves the SINR and reduces bit errors, but the effective throughput scales down as the number of parallel channels are reduced. If $T_{b l k}(\alpha, B)$ represents the MIMO capacity or maximum throughput of screen-camera communication for block-size $\mathrm{B}$, at distortion factor $\alpha$, then

$$
T_{b l k}(\alpha, B)=\frac{W_{f p s}}{k}\left(\frac{\alpha\left\|R_{c a m}\right\|}{B}\right) \log _{2}\left(1+\operatorname{SINR}_{b l k}(\alpha, B)\right),
$$

where $\frac{\alpha\left\|R_{\text {cam }}\right\|}{B}$ represents the number of parallel channels for multiplexing, and $S I N R_{b l k}(\alpha, B)$ is from equation (5). The factor $k$ implies that a minimum of $k$ temporal samples of the camera pixel are required for reliable decoding. In practice, to minimize detection and decoding errors, the camera framerate has to be synchronized with the modulation rate of pixel intensities on the screen as well as the refresh rate of the screen (typically $120 \mathrm{~Hz}$ ). Synchronization of cameras for communication is challenging due to the jittery nature (owing to software limitations and hardware design errors) of the frame-sampling using CMOS sensors that are widely used in mobile devices today.

\section{EXPERIMENTAL CALIBRATION AND VALIDATION}

In this section we describe the experiments we conducted to validate our screen-camera channel model. Since channel capacity cannot be measured directly, we estimate capacity indirectly by substituting the measured SINR, perspective distortion factor $\alpha$, and AWGN noise $\sigma_{n}^{2}$ into the analytical

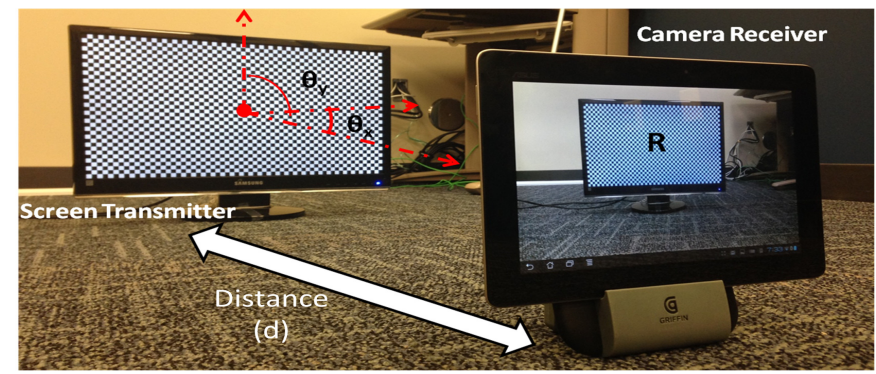

Fig. 4. Experiment setup showing LCD screen displaying black and white blocks of $B=60 \times 60$ pixels each

capacity expression derived in (6). We also show effect of perspective (position and orientation) and MIMO design parameters such as screen pixel block-size on the throughput of camera communication, by plotting the capacity estimates for the specific parameter choices. We then compare our capacity estimates with the throughput achieved by existing screencamera communication prototypes.

\section{A. General Experiment Methodology}

The experiment setup, as shown in Fig. 4, consisted of a 21.5inch Samsung LCD screen monitor of resolution $R_{s}=$ $1920 \times 1080$ pixels, that served as the screen-transmitter, and a 8MP camera of a ASUS Transformer Prime tablet (that ran Android OS version 4.1), that served as the camera receiver. The camera was operated at a resolution of $R_{\text {cam }}=1920 \times 1080$ and with no image compression. Exposure setting and white-balancing on the camera were set to auto (default setting in Android devices). Environment lighting conditions were the same for all our experiments. All our measurements were taken indoors in a lab-conference room setting equipped with fluorescent ceiling lighting. We fixed the screen and tablet onto stands so as to ensure the least amount of error in the measurement of distance and angle between the tablet and camera image planes. The raw dataset for our analysis consisted of image snapshots of the screen, displaying a chessboard pattern (blocks of B pixels each), captured by the tablet's camera at resolution of $R_{\text {cam }}$ pixels using a standard Android image capture application. The pixelintensity of a white block was set to 255 and the black at $25^{4}$ on the screen (the average intensity $P_{a v g}=140$ ). The image datasets consisted of 100 snapshots of the screen displaying the chessboard pattern, with the ceiling lights ON (an another dataset with lights OFF), at a set of distances, angles, and block-sizes. We changed angle between screen and camera by rotating the screen with respect to the $\mathrm{X}$ axis; distortions can be considered symmetrical on $\mathrm{X}$ and $\mathrm{Y}$ axis.

Camera Calibration: We obtained the the camera parameters, such as the focal length, pixel-side length, etc., through camera calibration procedure using the Caltech calibration toolbox [4], using which we determined the focal-distance to

\footnotetext{
${ }^{4}$ Due to the screen's residual back-lighting, intensities in [0,25] range did not cause any change in screen brightness
} 


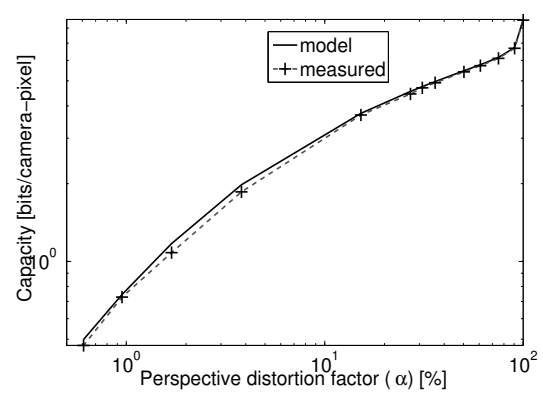

(a)

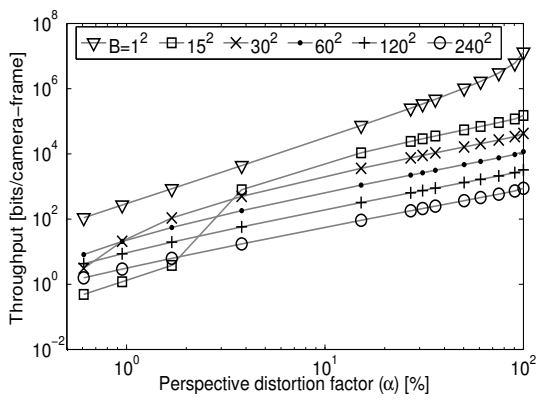

(b)

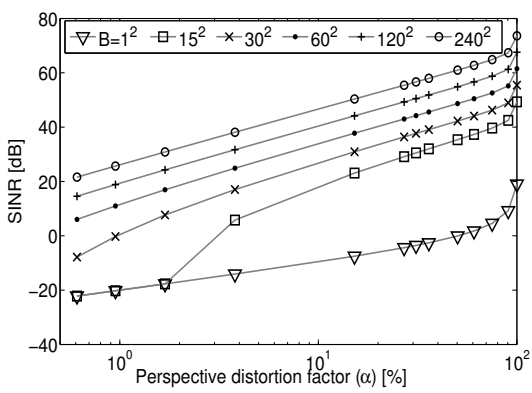

(c)

Fig. 5. (a) Capacity in bits/camera pixel $\left(C_{\text {campixel }}(\alpha)\right)$ for different perspective scaling $(\alpha)$ of screen image on camera (b) Throughput in bits/frame v/s $\alpha$ for different blocksizes ( 1 frame $=R_{\text {cam }}$ pixels, $B=15^{2}$ means $15 \times 15$ pixel block on screen) (c) SINR per block v/s $\alpha$ for different blocksizes B

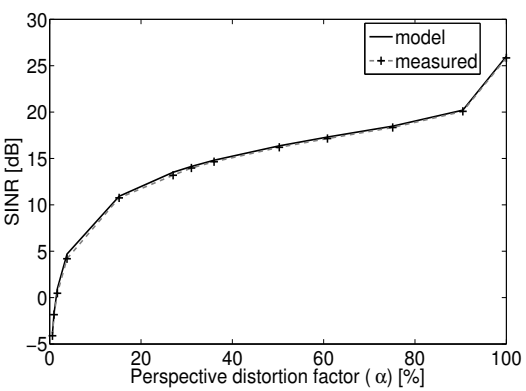

(a)

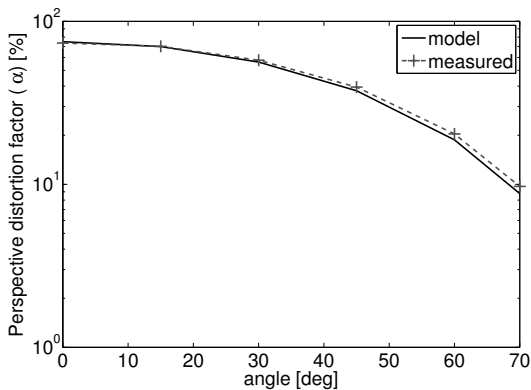

(b)

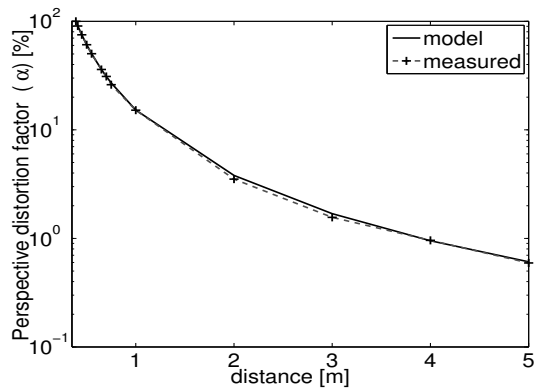

(c)

Fig. 6. (a) SINR for different perspective scaling $(\alpha)$ of screen image on camera (b) Perspective distortion $\alpha$ v/s angle between screen and camera (c) Perspective distortion factor $\alpha$ v/s distance between screen and camera

be $d_{f}=39 \mathrm{~cm}$. We also measured the blur variance $\sigma_{\text {blur }}^{2}$ by experimentally measuring the PSF of the tablet camera. The experiment involved emulating a point light source by illuminating one pixel on the LCD screen, and capturing its image from a distance of $d_{f}$ (so as to nullify any perspective scaling). Our results indicated that a Gaussian curve with a variance $\sigma_{b l u r}^{2}=0.25$ was the best fit to our measurements on each dimension. Table II summarizes the list of measured parameters from our experiments, along with the screen and camera specifications.

\section{B. Channel Capacity}

We estimate capacity of screen-camera channel by substituting the measured values of $S I N R_{\alpha}$, perspective distortion factor $\alpha$, and noise variance $\sigma_{n}^{2}$ in equation 6 . We evaluate capacity in bits per camera pixel as $C_{\text {campixel }}(\alpha)=\frac{C_{\text {cam }}(\alpha)}{\frac{W_{s}}{3}\left\|R_{\text {cam }}\right\|}$.

The measurement procedure for $\alpha, S I N R_{\alpha}, \sigma_{n}^{2}$ are explained in detail in sections V-C, V-D , and V-E respectively.

(1) Capacity v/s Perspective distortion factor: We plot the measured capacity in bits/camera-pixels for different perspective distortion factor values in Fig. 5 (a). The distortion factor $\alpha$ on the $\mathrm{x}$-axis is comprehensive of those obtained for each distance and angle combination. Fig. 5 (a) shows the our estimate from measurements fit well with the model (maximum error margin of 3\%). Fig. 5 (a) also shows that capacity depends significantly on the perspective of the camera, which is unique to camera channels, and unlike radio channels where multipath-fading plays a more significant role. We can observe that, about 1bit/camera pixel is achievable even when the screen is perspectively scaled onto only $15 \%$ on each dimension $(\alpha=2 \%)$ of the camera image. For the LCD-tablet system we used, this translates to a distance of $2.6 \mathrm{~m}\left(\frac{d_{f}}{0.15}\right)$. At a sampling rate of $30 \mathrm{fps}^{5}$ and at a resolution of $1920 \times 1080$, a data-rate of $31 \mathrm{Mbps}$ is achievable from an average-sized LCD monitor and a tablet camera. Assuming all parameters are the same, except the size of the screen is doubled, the same data-rate can be achieved at twice the range. Such data-rates are even sufficient for streaming applications, perhaps, to even download a video.

(2) Throughput with Block-size: We plot the screen-camera communication throughput from equation 7 in bits-per-frame $\left(\frac{T_{b l k}(\alpha, B)}{k W_{f p s}}\right)$ for different values of perspective distortion factors, and block sizes B, in Fig. 5 (b). We can observe from Fig. 5 (b) that capacity falls of steeply as $\alpha$ becomes smaller for smaller block-sizes; for example, at $B=15^{2}$ and $30^{2}$. The trend can be attributed to the low SINR at those perspectives as IPI increases due to the dense arrangement of bits (pixels carrying unique information). A block-size of 1 does not follow this trend as the gain due to capacity scaling due to more number of parallel channels compensates for most of the loss in SINR, however, trades-off with receiver complexity

\footnotetext{
${ }^{5}$ Typical frame-rate on smartphone/tablet cameras is $30 \mathrm{fps}$. IPhone $5 \mathrm{~S}$ has a 120fps capability [5]
} 
to detect the very low SINR signal. The trend in Fig. 5 (b) indicates that, while small block-size can yield considerable throughput at small distance (or angles), it is judicious to switch to a larger block-size at farther distance (or angle), and if such adaptations are not possible then it is judicious to use a globally optimal (determined through calibration) blocksize. For example, for the block-sizes in Fig. 5 (b) $B=30^{2}$ looks close to optimal.

(3) Comparison with Prototypes: We compare our MIMO capacity estimates $\left(T_{b l k}(\alpha, B)\right)$ with the throughput of existing prototypes of screen-camera communication. In PixNet [20], bits are modulated onto LCD screen pixels that are decoded by an off-the shelf point and shoot camera. PixNet uses OFDM for modulation and adds $(255,243)$ reed-solomon coding for error correction. Consistent with the definition of a block in our model, PixNet uses a block-size of $84 \times 84$. PixNet was evaluated using a 30inch LCD screen as the transmitter and $6 \mathrm{MP}$ CCD camera at the receiver, and up-to a maximum distance of $14 \mathrm{~m}$. The authors also reported the throughput from their implementation of QR codes, which we will call QR-P. The QR-P uses a version 5 QR code with a block size of $5 \times 5$ pixels, and that encodes 864 bits per QR code. On the other hand, COBRA [14] uses color barcodes to communicate between smartphone screen and camera, and was evaluated up-to a maximum distance of $22 \mathrm{~cm}$, and with a blocksize of $6 \times 6$ pixels. The authors of [14] have also implemented a smartphone (receiver) version of PixNet, which we will call PixNet-C, where the settings remained the same as original PixNet system.

\begin{tabular}{|cccc|}
\hline COBRA & PixNet-C & PixNet & QR-P \\
\hline $4.5 \mathrm{x}$ & $3 \mathrm{x}$ & $2.5 \mathrm{x}$ & $7 \mathrm{x}$ \\
\hline
\end{tabular}

TABLE I

RATIO OF CAPACITY OVER EXISTING PROTOTYPE'S THROUGHPUT ( $3 X$ INDICATES THE EXISTING PROTOTYPE IS 1/3RD OF CAPACITY)

In table I, we report the ratio of throughput from equation 7 to the throughput of the these prototypes, for the same parameter settings, of blocksize and $\alpha$ as in their existing implementations. Our estimates indicate that there is room for atleast $2.5 \mathrm{x}$ improvement in throughput when compared to capacity. The discrepancy in throughput in these existing prototypes can be attributed to different parameter choices. For example, PixNet uses OFDM modulation and coding which add communication overheads, which have to be incorporated in a limited spatial bandwidth available on the screen. COBRA also incurs loss in throughput due to coding overheads, and additionally the small block size allows for more interference, reducing SINR. COBRA minimizes blur by using repetitive colour patterns and intelligent placement of those patterns on the screen. While this strategy minimizes the effect of interference from neighboring pixels, the repetition causes under-utilization of the spatial bandwidth. In general, our findings, supported by these exemplar comparisons, open up interesting questions in the design space for improving information throughputs of screen-camera communication systems.

\section{Perspective Distortion Factor}

The objective of this experiment was to determine the perspective distortion factor $\alpha$ from our measurements to estimate capacity. Since $\alpha$ quantifies the relative area occupancy of the screen in the camera image, we measured the average distortion factor as,

$$
\alpha_{m}=\frac{\|R\|}{\left\|R_{\text {cam }}\right\|} \frac{1}{\left(1+4 \sigma_{\text {blur }}^{2}\right)}
$$

where $\|R\|$ represents to the total number of camera pixels that correspond to the imaged screen pixels, and $R_{\text {cam }}$ is the resolution of the camera. In figures 6(b) and 6(c) we plot $\alpha_{m}$ as a function of angle and distance, respectively. As can be seen from these plots the measured spatial-bandwidth fits well with the model (maximum error margin of $1.5 \%$ ). The $\alpha_{m}$ reported here is the perspective distortion factor for our LCD - tablet (camera) channel. The distance and angle at which $\alpha_{m}=0$ in these plots can be construed as the communication range of a system with the same screen and camera parameters. For example, for a screen with 10x the size (a billboard [2]) the distance range is close to $10 \mathrm{x}$ (about 40m) that of our experimental system.

\section{Signal-to-Interference Noise Ratio}

To facilitate capacity estimation, we measured the signalto-interference noise ratio $S I N R_{\alpha m e a s}$ in our experimental system. We will now discuss the measurement procedure in detail. Let $W_{i O N}(x, y)$ and $W_{i O F F}(x, y)$ represent the intensity of a pixel from a white block at location $(x, y)$ on the camera image where the lights were $\mathrm{ON}$ and $\mathrm{OFF}$ respectively, and $i(i=1,2 \ldots 100)$ being the index of the image in the dataset (similarly, $B_{i O N}(x, y)$ and $B_{i O F F}(x, y)$ represent pixel intensities from a black block). Let $S I N R_{W}$ denote the signal to interference noise ratio for the white pixel and $S I N R_{B}$ for the black, then

$$
\begin{aligned}
& S I N R_{\alpha \text { meas }}=\frac{1}{2} \sum\left(\frac{S I N R_{W}}{\|W\|}+\frac{S I N R_{B}}{\|B\|}\right) \\
& S I N R_{W}=\gamma_{1 m} \frac{s(W)}{k(B)+n(W)}+\gamma_{2 m} \frac{s(W)}{n(W)} \\
& S I N R_{B}=\gamma_{1 m} \frac{s(B)}{k(W)+n(B)}+\gamma_{2 m} \frac{s(B)}{n(B)} \\
& s(W)=\frac{1}{100} \sum_{i=1}^{100} \sum_{x, y}\left(\alpha_{m} W_{i O N}(x, y)\right)^{2} \\
& k(B)=\frac{1}{100} \sum_{i=1}^{100} \sum_{x^{\prime}, y^{\prime}}\left(1-\alpha_{m}\right)\left(B_{i O F F}\left(x^{\prime}, y^{\prime}\right)\right)^{2} \\
& n(W)=\frac{1}{100} \sum_{i=1}^{100} \sum_{x, y}\left(W_{i O N}(x, y)-W_{i O F F}(x, y)\right)^{2}
\end{aligned}
$$




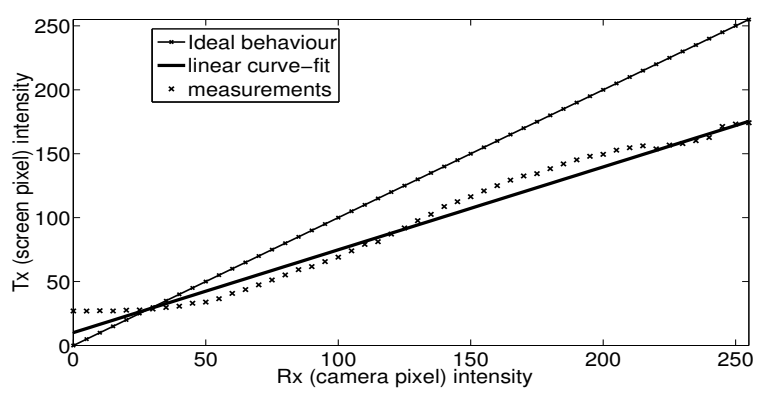

Fig. 7. Screen-Camera mapping

where $\left(x^{\prime}, y^{\prime}\right) \neq(x, y),\|W\|$ and $\|B\|$ represent the total number of white and black blocks respectively. $\gamma_{1 m}$ and $\gamma_{2 m}$ represent the measured number of pixels on the boundary and non-boundary blocks of the imaged block respectively.

We plot $S I N R_{\alpha m e a s}$ (from equation. (9)) versus $\alpha$, along with the analytical $S I N R_{\alpha}$ from equation (5), in Fig. 6 (a). We can observe from that our SINR measurements are in close agreement with our model (maximum error margin of $1.5 \mathrm{~dB}$ ). We plot the per-block measured SINR $\left(\operatorname{SINR}_{b l k}(\alpha, B)\right.$ using $\left.S I N R_{\text {ameas }}\right)$ versus $\alpha$ for different block-sizes B in Fig. 5 (c).

We can infer from Fig. 5 (c) that, larger the block higher is the the per-block SINR. We can also observe that for a block-size $B=1$, though it provides large number of parallel channels for multiplexing, the signal energy on each channel is much lower than the noise level, even for medium values of $\alpha$. In this case, additional signal processing is necessary at the receiver can help decode the low SINR signal with minimal errors. In general, the choice on the size of blocks becomes a primary design parameter as it affects SINR performance.

\section{E. Noise Measurement}

We empirically measured noise power for SINR computation, to aid analytical capacity estimation. The experiment dataset for this analysis consisted of 200 continuous camera snapshots of the LCD screen at $2 \mathrm{~m}$ (and perfect alignment), displaying gray-level intensities from 0-255 in steps of 5 (total 52 sets). Based on our measurements we realized that the intensity mapping between screen and camera can be linear approximated(as shown in Fig. 7) and can be numerically expressed as $g(x)=0.6481 x+10.06$ where $x=0,1, \ldots 255$, and the constant 10.06 accounts for the deterministic DC noise in the pixel. The factor 0.6481 can be treated as the path loss factor analogous to RF. As mentioned earlier, the AWGN noise from the background manifests as the temporal variance in the pixel intensity. We compute the noise energy per pixel in our LCD screen- tablet camera channel, using the mean-variance (vâr $r(g(x)$ : averaged over 52 samples) of the intensity mapping between the screen's actual intensity and the measured intensity on the camera pixel as, $\sigma_{n}^{2}=$ $10.06^{2}+\operatorname{vâ} r(g(x))=101.28$.

\begin{tabular}{|l|l|}
\hline Parameter & Value \\
\hline Cam pixel side-length $s_{\text {cam }}[\mu \mathrm{m}]$ & 65 \\
\hline Cam focal length $f_{\text {cam }}\left[\times s_{\text {cam }}\right]$ & 1573 \\
\hline Screen pixel side-length $s_{t}[\mathrm{~mm}]$ & 0.248 \\
\hline Principal point $\left(o_{x}, o_{y}\right)$ & $(960.1,539.2)$ \\
\hline Noise-variance $\sigma_{n}^{2}$ & 101.28 \\
\hline Lens-blur variance $\sigma_{\text {blur }}^{2}\left[\times s_{\text {cam }}^{2}\right]$ & 0.25 \\
\hline$\left\|R_{s}\right\|\left(=\left\|R_{\text {cam }}\right\|\right)[$ pixels $]$ & $1920 \times 1080$ \\
\hline Focal-distance $d_{f}[\mathrm{~m}]$ & 0.39 \\
\hline
\end{tabular}

TABLE II

TABLE OF SCREEN, CAMERA AND MEASURED PARAMETERS

\section{RELATED WORK}

Camera based communication is an example of visual MIMO communication [10] where camera is used as a receiver for information transmitted from arrays of light emitting elements. In our earlier work in [10] capacity of a camera channel was estimated by treating the transmitter light emitting array and the camera are perfectly aligned. The channel is considered as an analog communications channel where the signal at the receiver is the sampled photocurrents from each image pixel, and do not take into account the quantization limitations in the camera.

The LCD screen-camera channel capacity estimates [17] were based on a water-filing algorithm assuming the camera channel can be equalized to encounter the effects of spatial distortions. But the model and the prototype were designed for a fixed distance of $2 \mathrm{~m}$ between the screen and camera and did not study the effects of perspective on the estimated capacity and throughputs achieved. Perspective distortion has been studied by the imaging community previously [12], [22], but the fact that the camera is a part of a wireless communication channel (captured object is the light source itself) presents a new domain of challenge for applying imaging models in analyzing communication channels.

The advent of high-resolution cameras in mobile devices has spurred interest in using cameras for communication to retrieve information from screens [1], [14], [18], [20]. These applications use specific receiver processing schemes to combat visual distortions. PixNet [20] proposes to use OFDM modulation to combat the effect of perspective distortion on images by inverse filtering on the estimated channel, and using forward error correction. COBRA [14] proposes to leverage from encoding on the color channels to achieve throughput gains for smartphone screen-camera communication, but at very short distances $(22 \mathrm{~cm})$. The fact that several prototypes have been constructed reveals that screen-camera communication is gaining large momentum.

\section{CONCLUSION}

In this paper, we discussed the applicability of cameras for communication. We considered the example where cameras could be used as receivers for data transmitted in the form of time-varying 2D barcodes from display screens. We modeled a 
screen-camera channel using camera projection theory, which addressed visual channel perspective distortions in more detail than prior works. We discussed and modeled the effect of perspective distortion on the information capacity of screencamera communications, and validated the same through calibration experiments. Our capacity estimates indicated that, even with the frame-rate limitations in off-the-shelf mobile cameras, data-rates of the order of hundreds of kbps to Mbps is possible even when the $2 \mathrm{D}$ barcode from the screen images onto only a small portion of the camera image. Our findings indicated that camera communications is still promising for medium sized data-transfer or even streaming applications; such as downloading a file from a smartphone screen or streaming a movie from a large display wall. Our estimates indicate that current prototypes have only achieved less than half their capacity, which means that designing efficient techniques to address perspective distortions is still an open problem for building high-data rate camera communications.

\section{ACKNOWLEDGMENTS}

This work is supported by the US National Science Foundation (NSF) under the grant CNS-1065463

\section{REFERENCES}

[1] HCCB High Capacity Color Barcodes http://research.microsoft.com/enus/projects/hccb/about.aspx

[2] Billboard sizes. http://www.sbuilts.com/sizes.cfm.

[3] IEEE P802.15 Working Group for Wireless Personal Area Networks: On Study Group Status for Camera Communications. http://tinyurl.com/odkrr9w.

[4] Camera calibration toolbox for MATLAB. http://www.vision.caltech.edu/bouguetj/

[5] Apple-IPhone 5s-iSight Camera. http://www.apple.com/iphone-5s/camera/.

[6] Lecture notes by D.V. Valen: Point Spread Function Workshop http://tinyurl.com/p88lkbr.

[7] A. Goldsmith. Wireless Communications. Cambridge, 2005.

[8] Visible light communication consortium. http://vlcc.net.

[9] M. Varga, A. Ashok, W. Yuan, M. Gruteser, N. Mandayam ,and K. Dana. Demo: Visual MIMO based LED - Camera Communication Applied to Automobile Safety. In Proceedings of Mobisys, 2011.

[10] A. Ashok, M. Gruteser, N. Mandayam, J. Silva, M. Varga, and K. Dana. Challenge: Mobile Optical Networks Through Visual MIMO. In Proceedings of MobiCom, pages 105-112, New York, NY, USA, 2010. ACM.

[11] L. Zheng and D.N.C Tse, Diversity and Multiplexing: A Fundamental Tradeoff In Multiple-Antenna Channels In IEEE Transactions on Information Theory, vol. 49, 2003, pages 1073-1096

[12] H. Chen, R. Sukhthankar, G. Wallace, and T. jen Cham. Calibrating Scalable Multi-projector Displays Using Camera Homography Trees. In In CVPR, pages 9-14,2001.

[13] R. C. Gonzalez and R. E. Woods. Digital Image Processing. Prentice Hall, January 2002.

[14] T. Hao, R. Zhou, and G. Xing. Cobra: color barcode streaming for smartphone systems. In Proceedings of MobiSys '12, pages 85-98, New York, NY, USA, 2012. ACM.

[15] R. I. Hartley and A. Zisserman. Multiple View Geometry in Computer Vision. Cambridge University Press, ISBN: 0521540518, second edition, 2004

[16] B. K. P. Horn. Robot vision. MIT Press, Cambridge, MA, USA, 1986

[17] S. Hranilovic and F. Kschischang. A Pixelated-MIMO Wireless Optical Communication System. Selected Topics in Quantum Electronics, IEEE Journal of, 12(4):859 -874 , jul. 2006

[18] X. Liu, D. Doermann, and H. Li. A camera-based mobile data channel: capacity and analysis. In Proceedings of MM, pages 359-368, NY, USA, 2008. ACM

[19] T.S. Lomheim and G.C. Holst. CMOS/CCD sensors and camera systems. The International Society for Optical Engineering (SPIE), 2 edition, 2011

[20] S. D. Perli, N. Ahmed, and D. Katabi. PixNet: Interference-Free Wireless Links using LCD-Camera Pairs. In Proceedings of MobiCom '10, pages 137-148, New York, NY, USA, 2010. ACM.

[21] A. Tang, J. Kahn, and K.-P. Ho. Wireless Infrared Communication Links using Multi-beam Transmitters and Imaging Receivers. In ICC 96, Conference Record, Converging Technologies for Tomorrow's Applications, volume 1, pages 180-186 vol.1, Jun 1996.

[22] R. Yang, D. Gotz, J. Hensley, H. Towles, and M. S. Brown. Pixelflex: A Reconfigurable Multi-projector Display System, 2001.

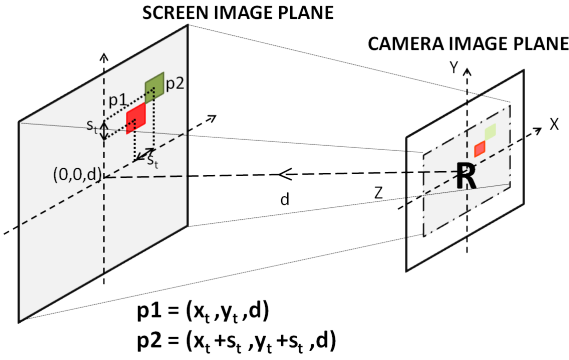

Fig. 8. Illustration Showing the Screen and Camera Image Axis (observe that, rotation about $\mathrm{Z}$ axis will not cause pixel distortion)

[23] T. Komine and M. Nakagawa. Fundamental Analysis for Visible-Light Communication System using LED Lights. IEEE Transactions on Consumer Electronics, 50(1):100-107, Feb 2004

\section{APPENDIX}

A. Derivation For Perspective Scaling Factor $\alpha_{p}$ Using Camera Projection Theory

Consider a point $\left[X_{w}, Y_{w}, Z_{w}\right]^{T}$ in world 3D space coordinates with respect to the camera image axis. The camera image $2 \mathrm{D}$ coordinates $[x, y]^{T}$ are given as,

$$
\left[\begin{array}{lll}
x & y & 1
\end{array}\right]^{T}=\mathbf{C}\left[\begin{array}{ll}
\mathbf{R} & \mathbf{T}
\end{array}\right]\left[\begin{array}{lll}
X_{w} & Y_{w} & Z_{w}
\end{array}\right]^{T}
$$

where $T$ denotes transpose operation, $\mathbf{C}, \mathbf{R}, \mathbf{T}$ are the camera calibration matrix, rotation matrix and translation vector respectively. Camera calibration matrix $\mathbf{C}$ accounts for the projection and scaling of the coordinates in the image $\left(\left(o_{x}, o_{y}\right)\right.$ is image center). $\mathbf{R}$ is the rotation matrix that accounts for the 3-tuple rotation angle $\left(\theta_{x}, \theta_{y}, \theta_{z}\right)$. and $\mathbf{T}$ accounts for the translation between the world coordinate and the camera axis. If $c \theta=\cos \theta, s \theta=\sin \theta$ then,

$$
\begin{gathered}
\mathbf{R}=\left[\begin{array}{ccc}
c \theta_{z} & -s \theta_{z} & 0 \\
s \theta_{z} & c \theta_{z} & 0 \\
0 & 0 & 1
\end{array}\right]\left[\begin{array}{ccc}
c \theta_{y} & 0 & s \theta_{y} \\
0 & 1 & 0 \\
-s \theta_{y} & 0 & c \theta_{y}
\end{array}\right]\left[\begin{array}{ccc}
1 & 0 & 0 \\
0 & c \theta_{x} & -s \theta_{x} \\
0 & s \theta_{x} & c \theta_{x}
\end{array}\right] \\
\mathbf{C}=\left[\begin{array}{ccc}
\frac{f}{s_{c a m}} & 0 & o_{x} \\
0 & \frac{f}{s_{c a m}} & o_{y} \\
0 & 0 & 1
\end{array}\right] \quad \mathbf{T}=\left[\begin{array}{c}
T_{w}^{x} \\
T_{w}^{y} \\
T_{w}^{z}
\end{array}\right]
\end{gathered}
$$

Consider two adjacent pixels p1 and p2 (of side-length $s_{t}$ ) of the screen transmitter situated at distance $d$ from the camera, as shown in Figure 8 . Let $x_{t}, y_{t}$ denote the distance of pixel $\mathrm{p} 1$ from the screen's center in $\mathrm{X}$ and $\mathrm{Y}$ dimensions respectively. Then using camera projection matrix equation from equation 10, the distortion in each pixel, $\alpha_{\left(x_{t}, y_{t}\right)}(x, y)$ can be derived as,

$$
\begin{gathered}
{\left[\begin{array}{c}
x_{p 1} \\
y_{p 1} \\
1
\end{array}\right]=\mathbf{C}[\mathbf{R} \mathbf{T}]\left[\begin{array}{c}
x_{t} \\
y_{t} \\
d
\end{array}\right]\left[\begin{array}{c}
x_{p 2} \\
y_{p 2} \\
1
\end{array}\right]=\mathbf{C}[\mathbf{R} \mathbf{T}]\left[\begin{array}{c}
x_{t}+s_{t} \\
y_{t}+s_{t} \\
d
\end{array}\right]} \\
\begin{aligned}
& \alpha_{\left(x_{t}, y_{t}\right)}(x, y)=\left|x_{p 2}-x_{p 1}\right| \times\left|y_{p 2}-y_{p 1}\right| \quad \forall(x, y) \in \mathbb{R} \\
&=0, \quad \text { otherwise } \\
& \alpha_{\left(x_{t}, y_{t}\right)}(x, y)=s_{t} \frac{\frac{f_{c a m}}{s_{c a m}}\left(c \theta_{y}+s \theta_{x} s \theta_{y}\right)+o_{x}\left(s \theta_{y}-s \theta_{x} c \theta_{y}\right)}{x_{t} s \theta_{y}-y_{t} s \theta_{x} c \theta_{y}+c \theta_{x} c \theta_{y} d} \\
& \times s_{t} \frac{\frac{f_{c a m}}{s_{c a m}}\left(c \theta_{y}\right)+o_{y}\left(s \theta_{y}-s \theta_{x} c \theta_{y}\right)}{x_{t} s \theta_{y}-y_{t} s \theta_{x} c \theta_{y}+c \theta_{x} c \theta_{y} d}
\end{aligned}
\end{gathered}
$$

where $|$.$| denotes the absolute value. \mathbb{R}$ denotes the set of camera pixels corresponding to the screen's projected image. We assumed that, $s_{t}$ (order of microns) « $d$ (order of $\mathrm{cm}$ or $\mathrm{m}$ ) in our derivation.

Using equation 15 the average distortion factor $\alpha_{p}$ can be determined as,

$$
\alpha_{p}=\frac{1}{\left\|R_{s}\right\|} \sum_{\forall\left(x_{t}, y_{t}\right)} \frac{1}{\left\|R_{c a m}\right\|} \sum_{\forall(x, y)} \alpha_{\left(x_{t}, y_{t}\right)}(x, y)
$$

where $\left\|R_{s}\right\|,\left\|R_{\text {cam }}\right\|$ are the screen and camera resolutions respectively. 